\title{
Generalization of the space $l(p)$ derived by absolute Euler summability and matrix operators
}

\author{
Fadime Gökçe ${ }^{1^{*}}$ (D) and Mehmet Ali Sarıöl ${ }^{1}$
}

\section{${ }^{*}$ Correspondence:} fgokce@pau.edu.tr

'Department of Mathematics, University of Pamukkale, Denizli, Turkey

\begin{abstract}
The sequence space /( $p$ ) having an important role in summability theory was defined and studied by Maddox (Q. J. Math. 18:345-355, 1967). In the present paper, we generalize the space $/(p)$ to the space $\left|E_{\phi}^{r}\right|(p)$ derived by the absolute summability of Euler mean. Also, we show that it is a paranormed space and linearly isomorphic to I(p). Further, we determine $\alpha$-, $\beta$-, and $\gamma$-duals of this space and construct its Schauder basis. Also, we characterize certain matrix operators on the space.
\end{abstract}

MSC: $40 \mathrm{CO} 05 ; 40 \mathrm{~F} 05 ; 46 \mathrm{~A} 45$

Keywords: Absolute summability; Euler means; Matrix transformations; Sequences spaces

\section{Introduction}

Let $X, Y$ be any subsets of $\omega$, the set of all sequences of complex numbers, and $A=\left(a_{n v}\right)$ be an infinite matrix of complex numbers. By $A(x)=\left(A_{n}(x)\right)$, we indicate the $A$-transform of a sequence $x=\left(x_{v}\right)$ if the series

$$
A_{n}(x)=\sum_{v=0}^{\infty} a_{n v} x_{v}
$$

are convergent for $n \geq 0$. If $A x \in Y$, whenever $x \in X$, then $A$, denoted by $A: X \rightarrow Y$, is called a matrix transformation from $X$ into $Y$, and we mean the class of all infinite matrices $A$ such that $A: X \rightarrow Y$ by $(X, Y)$. For $c_{s}, b_{s}$, and $l_{p}(p \geq 1)$, we write the space of all convergent, bounded, $p$-absolutely convergent series, respectively. Further, the matrix domain of an infinite matrix $A$ in a sequence space $X$ is defined by

$$
X_{A}=\left\{x=\left(x_{n}\right) \in \omega: A(x) \in X\right\}
$$

The $\alpha$-, $\beta$-, and $\gamma$-duals of the space $X$ are defined as follows:

$$
\begin{aligned}
& X^{\alpha}=\left\{\epsilon \in \omega:\left(\epsilon_{n} x_{n}\right) \in l_{1} \text { for all } x \in X\right\}, \\
& X^{\beta}=\left\{\epsilon \in \omega:\left(\epsilon_{n} x_{n}\right) \in c_{s} \text { for all } x \in X\right\}, \\
& X^{\gamma}=\left\{\epsilon \in \omega:\left(\epsilon_{n} x_{n}\right) \in b_{s} \text { for all } x \in X\right\} .
\end{aligned}
$$

(c) The Author(s) 2018. This article is distributed under the terms of the Creative Commons Attribution 4.0 International License (http://creativecommons.org/licenses/by/4.0/), which permits unrestricted use, distribution, and reproduction in any medium, provided you give appropriate credit to the original author(s) and the source, provide a link to the Creative Commons license, and indicate if changes were made. 
A subspace $X$ is called an $F K$ space if it is a Frechet space, that is, a complete locally convex linear metric space, with continuous coordinates $P_{n}: X \rightarrow C(n=1,2, \ldots)$, where $P_{n}(x)=x_{n}$ for all $x \in X$; an $F K$ space whose metric is given by a norm is said to be a $B K$ space. An $F K$ space $X$ including the set of all finite sequences is said to have $A K$ if

$$
\lim _{m \rightarrow \infty} x^{[m]}=\lim _{m \rightarrow \infty} \sum_{\nu=0}^{m} x_{\nu} e^{(v)}=x
$$

for every sequence $x \in X$, where $e^{(v)}$ is a sequence whose only non-zero term is one in $v$ th place for $v \geq 0$. For example, it is well known that the Maddox space

$$
l(p)=\left\{x=\left(x_{n}\right): \sum_{n=1}^{\infty}\left|x_{n}\right|^{p_{n}}<\infty\right\}
$$

is an $F K$ space with $A K$ with respect to its natural paranorm

$$
g(x)=\left(\sum_{n=0}^{\infty}\left|x_{n}\right|^{p_{n}}\right)^{1 / M},
$$

where $M=\max \left\{1, \sup _{n} p_{n}\right\}$; also it is even a $B K$ space if $p_{n} \geq 1$ for all $n$ with respect to the norm

$$
\|x\|=\inf \left\{\delta>0: \sum_{n=0}^{\infty}\left|x_{n} / \delta\right|^{p_{n}} \leq 1\right\}
$$

([19-21, 29]).

Throughout this paper, we assume that $0<\inf p_{n} \leq H<\infty$ and $p_{n}^{*}$ is a conjugate of $p_{n}$, i.e., $1 / p_{n}+1 / p_{n}^{*}=1, p_{n}>1$, and $1 / p_{n}^{*}=0$ for $p_{n}=1$.

Let $\sum a_{v}$ be a given infinite series with $s_{n}$ as its $n$th partial sum, $\phi=\left(\phi_{n}\right)$ be a sequence of positive real numbers and $p=\left(p_{n}\right)$ be a bounded sequence of positive real numbers. The series $\sum a_{\nu}$ is said to be summable $\left|A, \phi_{n}\right|(p)$ if (see [10])

$$
\sum_{n=1}^{\infty}\left(\phi_{n}\right)^{p_{n}-1}\left|A_{n}(s)-A_{n-1}(s)\right|^{p_{n}}<\infty .
$$

It should be noted that the summability $\left|A, \phi_{n}\right|(p)$ includes some well-known summability methods for special cases of $A, \phi$ and $p=\left(p_{n}\right)$. For example, if we take $A=E^{r}$ and $p_{n}=k$ for all $n$, then it is reduced to the summability method $|E, r|_{k}$ (see [12]) where Euler matrix $E^{r}$ is defined by

$$
e_{n k}^{r}= \begin{cases}\left(\begin{array}{l}
n \\
k
\end{array}\right)(1-r)^{n-k} r^{k}, & 0 \leq k \leq n, \\
0, & k>n,\end{cases}
$$

for $0<r<1$ and

$$
e_{n k}^{1}= \begin{cases}0, & 0 \leq k<n \\ 1, & k=n\end{cases}
$$


Also we refer the readers to the papers [7, 9, 30, 31, 35] for detailed terminology.

A large literature body, concerned with producing sequence spaces by means of matrix domain of a special limitation method and studying their algebraic, topological structure and matrix transformations, has recently grown. In this context, the sequence spaces $\bar{l}(p)$, $r_{p}^{t}, l(u, v, p)$, and $l\left(N^{t}, p\right)$ were studied by Choudhary and Mishra [8], Altay and Başar [2, 3], Yeşilkayagil and Başar [37] by defining as the domains of the band, Riesz, the factorable, and Nörlund matrices in the $l(p)$ (see also $[1,4-6,16-18,23-28]$ ).

Also, some series spaces have been derived and examined by various absolute summability methods from a different point of view (see [13, 14, 32, 34]). In this paper, we generalize the space $l(p)$ to the space $\left|E_{\phi}^{r}\right|(p)$ derived by the absolute summability of Euler means and show that it is a paranormed space linearly isomorphic to $l(p)$. Further, we determine $\alpha$-, $\beta$-, and $\gamma$-duals of this space and construct its Schauder basis. Finally, we characterize certain matrix transformations on the space.

First, we remind some well-known lemmas which play important roles in our research.

\section{Needed lemmas}

Lemma 2.1 ([11]) Let $p=\left(p_{v}\right)$ and $q=\left(q_{v}\right)$ be any two bounded sequences of strictly positive numbers.

(i) If $p_{v}>1$ for all $v$, then $A \in\left(l(p), l_{1}\right)$ if and only if there exists an integer $M>1$ such that

$$
\sup \left\{\sum_{v=0}^{\infty}\left|\sum_{n \in K} a_{n v} M^{-1}\right|^{p_{v}^{*}}: K \subset N \text { finite }\right\}<\infty .
$$

(ii) If $p_{v} \leq 1$ and $q_{v} \geq 1$ for all $v \in N$, then $A \in(l(p), l(q))$ if and only if there exists some $M$ such that

$$
\sup _{v} \sum_{n=0}^{\infty}\left|a_{n v} M^{-1 / p_{v}}\right|^{q_{n}}<\infty .
$$

(iii) If $p_{v} \leq 1$, then $A \in(l(p), c)$ if and only if

$$
\text { (a) } \lim _{n} a_{n v} \text { exists for each } v, \quad \text { (b) } \sup _{n, v}\left|a_{n v}\right|^{p_{v}}<\infty \text {, }
$$

and $A \in\left(l(p), l_{\infty}\right)$ iff $(\mathrm{b})$ holds.

(iv) If $p_{v}>1$ for all $v$, then $A \in(l(p), c)$ if and only if (a) (a) holds, and (b) there is a number $M>1$ such that

$$
\sup _{n} \sum_{v=0}^{\infty}\left|a_{n v} M^{-1}\right|^{p_{v}^{*}}<\infty
$$

and $A \in\left(l(p), l_{\infty}\right)$ iff $(\mathrm{b})$ holds.

It may be noticed that condition (2) exposes a rather difficult condition in applications. The following lemma produces a condition to be equivalent to (2) and so the following lemma, which is more practical in many cases, will be used in the proofs of theorems. 
Lemma 2.2 ([33]) Let $A=\left(a_{n v}\right)$ be an infinite matrix with complex numbers and $\left(p_{v}\right)$ be a bounded sequence of positive numbers. If $U_{p}[A]<\infty$ or $L_{p}[A]<\infty$, then

$$
(2 C)^{-2} U_{p}[A] \leq L_{p}[A] \leq U_{p}[A]
$$

where $C=\max \left\{1,2^{H-1}\right\}, H=\sup _{v} p_{v}$,

$$
U_{p}[A]=\sum_{\nu=0}^{\infty}\left(\sum_{n=0}^{\infty}\left|a_{n v}\right|\right)^{p_{v}}
$$

and

$$
L_{p}[A]=\sup \left\{\sum_{\nu=0}^{\infty}\left|\sum_{n \in K} a_{n v}\right|^{p_{v}}: K \subset N \text { finite }\right\} .
$$

Lemma 2.3 ([22]) Let $X$ be an FK space with $A K, T$ be a triangle, $S$ be its inverse, and $Y$ be an arbitrary subset of $\omega$. Then we have $A \in\left(X_{T}, Y\right)$ if and only if $\widehat{A} \in(X, Y)$ and $V^{(n)} \in(X, c)$ for all $n$, where

$$
\hat{a}_{n v}=\sum_{j=v}^{\infty} a_{n j} j_{j v} ; \quad n, v=0,1, \ldots
$$

and

$$
v_{m v}^{(n)}= \begin{cases}\sum_{j=v}^{m} a_{n j} s_{j v}, & 0 \leq v \leq m \\ 0, & v>m .\end{cases}
$$

\section{Main theorems}

In this section, we introduce the paranormed series space $\left|E_{\phi}^{r}\right|(p)$ as the set of all series summable by the absolute summability method of Euler matrix and show that this space is linearly isomorphic to the space $l(p)$. Also, we compute the Schauder base, $\alpha-, \beta-$, and $\gamma$ duals of the space and characterize certain matrix transformations defined on that space. First of all, we note that, by the definition of the summability $\left|A, \phi_{n}\right|(p)$, we can write the space $\left|E_{\phi}^{r}\right|(p)$ as

$$
\left|E_{\phi}^{r}\right|(p)=\left\{a \in \omega: \sum_{n=0}^{\infty} \phi_{n}^{p_{n}-1}\left|\triangle A_{n}^{r}(s)\right|^{p_{n}}<\infty\right\},
$$

where

$$
\triangle A_{n}^{r}(s)=A_{n}^{r}(s)-A_{n-1}^{r}(s)
$$

and

$$
A_{n}^{r}(s)=\sum_{k=0}^{n}\left(\begin{array}{l}
n \\
k
\end{array}\right)(1-r)^{n-k} r^{k} s_{k}, \quad n \geq 0, \quad A_{-1}^{r}(s)=0 .
$$


Also, a few calculations give

$$
\begin{aligned}
\triangle A_{n}^{r}(s) & =\sum_{m=0}^{n} \sum_{k=m}^{n}\left(\begin{array}{l}
n \\
k
\end{array}\right)(1-r)^{n-k} r^{k} a_{m}-\sum_{m=0}^{n-1} \sum_{k=m}^{n-1}\left(\begin{array}{c}
n-1 \\
k
\end{array}\right)(1-r)^{n-1-k} r^{k} a_{m} \\
& =\sum_{m=1}^{n} \sum_{k=m}^{n}(1-r)^{n-1-k}\left[\left(\begin{array}{l}
n-1 \\
k-1
\end{array}\right)-r\left(\begin{array}{l}
n \\
k
\end{array}\right)\right] r^{k} a_{m} \\
& =\sum_{m=1}^{n} \sigma_{n m} a_{m}
\end{aligned}
$$

where

$$
\sigma_{n m}= \begin{cases}\sum_{k=m}^{n}(1-r)^{n-1-k} r^{k}\left[\left(\begin{array}{l}
n-1 \\
k-1
\end{array}\right)-r\left(\begin{array}{l}
n \\
k
\end{array}\right)\right], & 1 \leq m \leq n, \\
0, & m>n .\end{cases}
$$

Further, it follows by putting $r=q(1+q)^{-1}$

$$
\begin{aligned}
\sigma_{n m} & =(1+q)^{1-n} \sum_{k=m}^{n} q^{k}\left[\left(\begin{array}{l}
n-1 \\
k-1
\end{array}\right)-q(1+q)^{-1}\left(\begin{array}{l}
n \\
k
\end{array}\right)\right] \\
& =(1+q)^{-n} \sum_{k=m}^{n}\left[q^{k}\left(\begin{array}{l}
n-1 \\
k-1
\end{array}\right)-q^{k+1}\left(\begin{array}{c}
n-1 \\
k
\end{array}\right)\right] \\
& =q^{m}(1+q)^{-n}\left(\begin{array}{c}
n-1 \\
m-1
\end{array}\right)=\left(\begin{array}{c}
n-1 \\
m-1
\end{array}\right)(1-r)^{n-m} r^{m} .
\end{aligned}
$$

Now, by considering $T_{n}^{r}(\phi, p)(a)=\phi_{n}^{1 / p_{n}^{*}} \Delta A_{n}^{r}(s)$, we immediately get that $T_{0}^{r}(\phi, p)(a)=$ $a_{0} \phi_{0}^{1 / p_{0}^{*}}$ and

$$
\begin{aligned}
T_{n}^{r}(\phi, p)(a) & =\phi_{n}^{1 / p_{n}^{*}} \sum_{k=1}^{n}\left(\begin{array}{l}
n-1 \\
k-1
\end{array}\right)(1-r)^{n-k} r^{k} a_{k} \\
& =\sum_{k=1}^{n} t_{n k}^{r}(\phi, p) a_{k},
\end{aligned}
$$

where

$$
t_{n k}^{r}(\phi, p)= \begin{cases}\phi_{0}^{1 / p_{0}^{*}}, & k=n=0, \\
\phi_{n}^{1 / p_{n}^{*}}\left(\begin{array}{c}
n-1 \\
k-1
\end{array}\right)(1-r)^{n-k} r^{k}, & 1 \leq k \leq n, \\
0, & k>n .\end{cases}
$$

Therefore, we can state the space $\left|E_{\phi}^{r}\right|(p)$ as follows:

$$
\left|E_{\phi}^{r}\right|(p)=\left\{a=\left(a_{k}\right): \sum_{n=1}^{\infty}\left|\phi_{n}^{1 / p_{n}^{*}} \sum_{k=1}^{n}\left(\begin{array}{c}
n-1 \\
k-1
\end{array}\right)(1-r)^{n-k} r^{k} a_{k}\right|^{p_{n}}<\infty\right\},
$$


or

$$
\left|E_{\phi}^{r}\right|(p)=[l(p)]_{T^{r}(\phi, p)}
$$

according to notation (1).

Further, since every triangle matrix has a unique inverse which is a triangle (see [36]), the matrix $T^{r}(\phi, p)$ has a unique inverse $S^{r}(\phi, p)=\left(s_{n k}^{r}(\phi, p)\right)$ given by

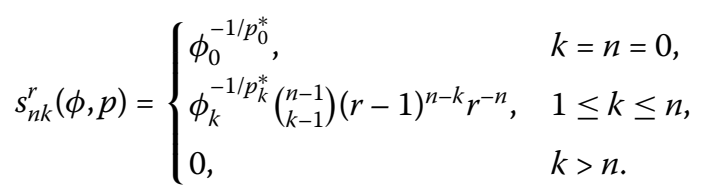

Before main theorems, note that if $r=1$ and $\phi_{n}=1$ for all $n \geq 0$, the space $\left|E_{\phi}^{r}\right|(p)$ is reduced to the space $l(p)$.

Theorem 3.1 Let $0<r<1$ and $p=\left(p_{n}\right)$ be a bounded sequence of non-negative numbers. Then:

(a) The set $\left|E_{\phi}^{r}\right|(p)$ becomes a linear space with the coordinate-wise addition and scalar multiplication, and also it is an FK-space with respect to the paranorm

$$
\|x\|_{\left|E_{\phi}^{r}\right|(p)}=\left(\sum_{n=0}^{\infty}\left|T_{n}^{r}(\phi, p)(x)\right|^{p_{n}}\right)^{1 / M}
$$

where $M=\max \left\{1, \sup p_{n}\right\}$.

(b) The space $\left|E_{\phi}^{r}\right|(p)$ is linearly isomorphic to the space $l(p)$, i.e., $\left|E_{\phi}^{r}\right|(p) \cong l(p)$.

(c) Define a sequence $\left(b_{n}^{(v)}\right)$ by $S^{r}\left(\left(e^{(v)}\right)\right)=\left(\sum_{v=0}^{n} s_{n v}^{r}(\phi, p) e^{(v)}\right)$. Then the sequence $\left(b_{n}^{(v)}\right)$ is the Schauder base of the space $\left|E_{\phi}^{r}\right|(p)$.

(d) The space $\left|E_{\phi}^{r}\right|(p)$ is separable.

Proof (a) The first part is a routine verification, so it is omitted. Since $T^{r}(\phi, p)$ is a triangle matrix and $l(p)$ is an $F K$-space, it follows from Theorem 4.3.2 in [36] that $\left|E_{\phi}^{r}\right|(p)=$ $[l(p)]_{T^{r}(\phi, p)}$ is an $F K$-space.

(b) We should show that there exists a linear bijection between the spaces $\left|E_{\phi}^{r}\right|(p)$ and $l(p)$. Now, consider $T^{r}(\phi, p):\left|E_{\phi}^{r}\right|(p) \rightarrow l(p)$ given by (3). Since the matrix corresponding this transformation is a triangle, it is obvious that $T^{r}(\phi, p)$ is a linear bijection. Furthermore, since $T^{r}(\phi, p)(x) \in l(p)$ for $x \in\left|E_{\phi}^{r}\right|(p)$, we get

$$
\|x\|_{\left|E_{\phi}^{r}\right|(p)}=\left(\sum_{n=0}^{\infty}\left|T_{n}^{r}(\phi, p)(x)\right|^{p_{n}}\right)^{1 / M}=\left\|T^{r}(\phi, p)(x)\right\|_{l(p)} .
$$

So, $T^{r}(\phi, p)$ preserves the paranorm, which completes this part of the proof.

(c) Since the sequence $\left(e^{(v)}\right)$ is the Schauder base of the space $l(p)$ and $\left|E_{\phi}^{r}\right|(p)=$ $[l(p)]_{T^{r}(\phi, p)}$, it can be written from Theorem 2.3 in [15] that $b^{(v)}=\left(S^{r}(\phi, p)\left(e^{(v)}\right)\right)$ is a Schauder base of the space $\left|E_{\phi}^{r}\right|(p)$.

(d) Since the space $\left|E_{\phi}^{r}\right|(p)$ is a linear metric space with a Schauder base, it is separable. 
Theorem 3.2 Let $0<r<1$. Define

$$
\begin{aligned}
& D_{1}^{r}=\left\{a \in \omega: \exists M>1, \sum_{v=0}^{\infty}\left(\sum_{n=v}^{\infty}\left|M^{-1} b_{n}^{(v)} a_{n}\right|\right)^{p_{v}^{*}}<\infty\right\}, \\
& D_{2}^{r}=\left\{a \in \omega: \exists M>1, \sup _{v} M^{1 / p_{v}} \sum_{n=v}^{\infty}\left|b_{n}^{(v)} a_{n}\right|<\infty\right\}, \\
& D_{3}^{r}=\left\{a \in \omega: \sum_{n=v}^{\infty} b_{n}^{(v)} a_{n} \text { converges for each } v\right\}, \\
& D_{4}^{r}=\left\{a \in \omega: \exists M>1, \sup _{n} \sum_{v=1}^{n}\left|\sum_{k=v}^{n} b_{k}^{(v)} a_{k} M^{-1}\right|<\infty\right\}, \\
& D_{5}^{r}=\left\{a \in \omega: \sup _{n, v}\left|\sum_{k=v}^{n} b_{k}^{(v)} a_{k}\right|^{p_{v}^{*}}<\infty\right\} .
\end{aligned}
$$

(i) If $p_{v}>1$ for all $v$, then

$$
\left\{\left|E_{\phi}^{r}\right|(p)\right\}^{\alpha}=D_{1}^{r}, \quad\left\{\left|E_{\phi}^{r}\right|(p)\right\}^{\beta}=D_{4}^{r} \cap D_{3}^{r}, \quad\left\{\left|E_{\phi}^{r}\right|(p)\right\}^{\gamma}=D_{4}^{r} .
$$

(ii) If $p_{v} \leq 1$ for all $v$, then

$$
\left\{\left|E_{\phi}^{r}\right|(p)\right\}^{\alpha}=D_{2}^{r}, \quad\left\{\left|E_{\phi}^{r}\right|(p)\right\}^{\beta}=D_{5}^{r} \cap D_{3}^{r}, \quad\left\{\left|E_{\phi}^{r}\right|(p)\right\}^{\gamma}=D_{5}^{r} .
$$

Proof To avoid the repetition of a similar statement, we only calculate $\beta$-duals of $\left|E_{\phi}^{r}\right|(p)$.

(i) Let us recall that $a \in\left\{\left|E_{\phi}^{r}\right|(p)\right\}^{\beta}$ if and only if $a x \in c s$ whenever $x \in\left|E_{\phi}^{r}\right|(p)$. Now, by using (5), it can be obtained that

$$
\begin{aligned}
\sum_{k=0}^{n} a_{k} x_{k} & =T_{0}^{r}(\phi, p)(x) \phi_{0}^{-1 / p_{0}^{*}} a_{0}+\sum_{k=1}^{n} a_{k} \sum_{v=1}^{k} \phi_{v}^{-1 / p_{v}^{*}}\left(\begin{array}{c}
k-1 \\
v-1
\end{array}\right)(r-1)^{k-v} r^{-k} T_{v}^{r}(\phi, p)(x) \\
& =T_{0}^{r}(\phi, p)(x) \phi_{0}^{-1 / p_{0}^{*}} a_{0}+\sum_{v=1}^{n} \phi_{v}^{-1 / p_{v}^{*}} T_{v}^{r}(\phi, p)(x) \sum_{k=v}^{n} a_{k}\left(\begin{array}{c}
k-1 \\
v-1
\end{array}\right)(r-1)^{k-v} r^{-k} \\
& =\sum_{v=0}^{n} d_{n v} T_{v}^{r}(\phi, p)(x),
\end{aligned}
$$

where $D=\left(d_{n v}\right)$ is defined by

$$
d_{n v}= \begin{cases}\phi_{0}^{-1 / p_{0}^{*}} a_{0}, & n=v=0, \\ \sum_{k=v}^{n} b_{k}^{(v)} a_{k}, & 1 \leq v \leq n, \\ 0, & v>n .\end{cases}
$$

Since $T^{r}(\phi, p)(x) \in l(p)$ whenever $x \in\left|E_{\phi}^{r}\right|(p), a \in\left\{\left|E_{\phi}^{r}\right|(p)\right\}^{\beta}$ if and only if $D \in(l(p), c)$. So, it follows from Lemma 2.1 that $a \in D_{4}^{r} \cap D_{3}^{r}$ if $p_{v}>1$ for all $v$, and also $a \in D_{5}^{r} \cap D_{3}^{r}$ if $p_{v} \leq 1$ for all $v$.

The remaining part of the theorem can be similarly proved by Lemma 2.1. 
Theorem 3.3 Let $A=\left(a_{n v}\right)$ be an infinite matrix of complex numbers, $\left(\phi_{n}\right)$ and $\left(\psi_{n}\right)$ be sequences of positive numbers, $p=\left(p_{n}\right)$ and $q=\left(q_{n}\right)$ be arbitrary bounded sequences of positive numbers with $p_{n} \leq 1$ and $q_{n} \geq 1$ for all $n$. Further, let the matrix $\hat{A}$ be defined by

$$
\hat{a}_{n v}=\sum_{j=v}^{\infty} a_{n j} b_{j}^{(v)}
$$

and $F=T^{r}(\psi, q) \hat{A}$. Then $A \in\left(\left|E_{\phi}^{r}\right|(p),\left|E_{\psi}^{r}\right|(q)\right)$ if and only if there exists an integer $M>1$ such that, for $n=0,1, \ldots$,

$$
\begin{aligned}
& \sum_{k=v}^{\infty} b_{k}^{(v)} a_{n k} \quad \text { converges for each } v, \\
& \sup _{m, v}\left|\sum_{k=v}^{m} b_{k}^{(v)} a_{n k}\right|^{p_{v}}<\infty
\end{aligned}
$$

and

$$
\sup _{v} \sum_{n=0}^{\infty}\left|M^{-1 / p_{v}} f_{n v}\right|^{q_{n}}<\infty
$$

Proof Suppose that $p_{v} \leq 1, q_{v} \geq 1$ for all $v$. Note that $\left|E_{\phi}^{r}\right|(p)=[l(p)]_{T^{r}(\phi, p)}$ and $\left|E_{\psi}^{r}\right|(q)=$ $[l(q)]_{T^{r}(\psi, q)}$. By Lemma 2.3, $A \in\left(\left|E_{\phi}^{r}\right|(p),\left|E_{\psi}^{r}\right|(q)\right)$ if and only if $\hat{A} \in\left(l(p),\left|E_{\psi}^{r}\right|(q)\right)$ and $V^{(n)} \in$ $(l(p), c)$, where the matrix $V^{(n)}$ is defined by

$$
v_{m \nu}^{(n)}= \begin{cases}\sum_{j=v}^{m} b_{j}^{(v)} a_{n j}, & 0 \leq v \leq m, \\ 0, & v>m .\end{cases}
$$

One can see that since $\hat{A}(x) \in\left|E_{\psi}^{r}\right|(q)=[l(q)]_{T^{r}(\psi, q)}$ whenever $x \in l(p), \hat{A} \in\left(l(p),\left|E_{\psi}^{r}\right|(q)\right)$ iff $F=T^{r}(\psi, q) \hat{A} \in(l(p), l(q))$. Now, applying Lemma 2.1(ii) and (iii) to the matrices $F$ and $V^{(n)}$, it follows that $V^{(n)} \in(l(p), c)$ iff, for $n=0,1, \ldots$, conditions (6) and (7) hold, and $F \in$ $(l(p), l(q))$ iff there exists an integer $M$ such that

$$
\sup _{v} \sum_{n=0}^{\infty}\left|M^{-1 / p_{v}} f_{n v}\right|^{q_{n}}<\infty
$$

which completes the proof.

Theorem 3.4 Assume that $A=\left(a_{n v}\right)$ is an infinite matrix of complex numbers and $\left(\phi_{n}\right)$, $\left(\psi_{n}\right)$ are sequences of positive numbers. If $p=\left(p_{n}\right)$ is an arbitrary bounded sequence of positive numbers such that $p_{n}>1$ for all $n$, and $H=T^{r}(\psi, 1) \hat{A}$, then $A \in\left(\left|E_{\phi}^{r}\right|(p),\left|E_{\psi}^{r}\right|(1)\right)$ if and only if there exists an integer $M>1$ such that, for $n=0,1, \ldots$,

$$
\begin{aligned}
& \sum_{k=v}^{\infty} b_{k}^{(v)} a_{n k} \text { converges for each } v \\
& \sup _{n} \sum_{v=0}^{\infty}\left|\sum_{k=v}^{n} b_{k}^{(v)} a_{n k} M^{-1}\right|^{p_{v}^{*}}<\infty
\end{aligned}
$$


and

$$
\sum_{v=0}^{\infty}\left(\sum_{n=0}^{\infty}\left|M^{-1} h_{n v}\right|\right)^{p_{v}^{*}}<\infty
$$

Proof Let $p_{n}>1$ for all $n$. It is clear that $\left|E_{\phi}^{r}\right|(p)=[l(p)]_{T^{r}(\phi, p)}$ and $\left|E_{\psi}^{r}\right|(1)=l_{T^{r}(\psi, 1)}$. So, by Lemma 2.3, we have $A \in\left(\left|E_{\phi}^{r}\right|(p),\left|E_{\psi}^{r}\right|(1)\right)$ if and only if $\hat{A} \in\left(l(p),\left|E_{\psi}^{r}\right|(1)\right)$ and $V^{(n)} \in$ $(l(p), c)$, where $\hat{A}$ and $V^{(n)}$ are given in Theorem 3.3. If we take $H=T^{r}(\psi, 1) \hat{A}$, then it is easily seen that $\hat{A} \in\left(l(p),\left|E_{\psi}^{r}\right|(1)\right)$ iff $H \in\left(l(p), l_{1}\right)$ because, if $\hat{A}(x) \in\left|E_{\psi}^{r}\right|(1)$ for all $x \in l_{1}(p)$, $H(x)=T^{r}(\psi, 1)(\hat{A}(x)) \in l_{1}$. So, applying Lemma 2.1 (iv) to the matrix $V^{(n)}$, it is obtained that $V^{(n)} \in(l(p), c)$ iff conditions (9) and (10) are satisfied. Again, if we apply Lemma 2.1(i) and Lemma 2.2 to the matrix $H$, then we have $H \in\left(l(p), l_{1}\right)$ iff the last condition holds.

\section{Conclusion}

The sequence spaces defined as domains of Riesz, factorable, Nörlund and $S$-matrices in the spaces $l(p)$ and the space of series summable by the absolute Euler have been recently studied by several authors. In this paper, we have defined the new absolute Euler space $\left|E_{\phi}^{r}\right|(p)$ and investigated some topological and algebraic properties such as isomorphism, duals, base, and also characterized certain matrix transformations on that space. So, we have extended some well-known results.

Acknowledgements

We thank the editor and referees for their careful reading, valuable suggestions and remarks.

Funding

No funding was received.

Competing interests

The authors declare that they have no competing interests.

Authors' contributions

Both authors contributed equally to the manuscript, read and approved the final manuscript.

\section{Publisher's Note}

Springer Nature remains neutral with regard to jurisdictional claims in published maps and institutional affiliations.

Received: 9 March 2018 Accepted: 7 June 2018 Published online: 15 June 2018

References

1. Alotaibi, A., Mursaleen, M., Alamri, B.A.S., Mohiuddine, S.A.: Compact operators on some Fibonacci difference sequence spaces. J. Inequal. Appl. 2015, 203 (2015)

2. Altay, B., Başar, F:: On the paranormed Riesz sequence spaces of non-absolute type. Southeast Asian Bull. Math. 26(5), 701-715 (2002)

3. Altay, B., Başar, F.: Generalization of the sequence space /(p) derived by weighted mean. J. Math. Anal. Appl. 330(1), 174-185 (2007)

4. Altay, B., Başar, F., Mursaleen, M.: On the Euler sequence spaces which include the spaces $I_{p}$ and $I_{\infty} I$. Inf. Sci. 176(10), 1450-1462 (2005)

5. Altay, B., Başar, F., Mursaleen, M.: On the Euler sequence spaces which include the spaces $I_{p}$ and $I_{\infty}$ II. Nonlinear Anal. 65(3), 707-717 (2006)

6. Başarır, M., Kara, E.E., Konca, Ş.: On some new weighted Euler sequence spaces and compact operators. Math. Inequal. Appl. 17(2), 649-664 (2014)

7. Bor, $H .:$ On $\left|\bar{N}, p_{n}\right|_{k}$ summability factors of infinite series. Tamkang J. Math. 16(1), 13-20 (1985)

8. Choudhary, B., Mishra, S.K.: A note on Köthe-Toeplitz duals of certain sequence spaces and their matrix transformations. Int. J. Math. Math. Sci. 18(4), 681-688 (1995)

9. Flett, T.M.: On an extension of absolute summability and some theorems of Littlewood and Paley. Proc. Lond. Math. Soc. 7, 113-141 (1957)

10. Gökçe, F., Sarıgöl, M.A.: Matrix operators on the series space $\left|\bar{N}_{p}^{\theta}\right|(\mu)$ and applications. Kuwait J. Sci. (in press)

11. Grosse-Erdmann, K.G.: Matrix transformations between the sequence spaces of Maddox. J. Math. Anal. Appl. 180 223-238 (1993) 
12. Hardy, G.H.: Divergent Series, vol. 334. Am. Math. Soc., Providence (2000)

13. Hazar, G.C., Gökçe, F.: Characterizations of matrix transformations on the series spaces derived by absolute factorable summability. In: Developments in Science and Engineering, pp. 411-426 (2016)

14. Hazar, G.C., Sarıgöl, M.A.: Compact and matrix operators on the space |C,-1| k. J. Comput. Anal. Appl. 25, 1014-1024 (2018)

15. Jarrah, A.M., Malkowsky, E.: Ordinary absolute and strong summability and matrix transformations. Filomat 17, 59-78 (2003)

16. Kara, E.E., Başarır, M.: On compact operators and some Euler $B^{(m)}$-difference sequence spaces. J. Math. Anal. Appl. 279(2), 459-511 (2011)

17. Kara, E.E., Ilkhan, M.: On some Banach sequence spaces derived by a new band matrix. Br. J. Math. Comput. Sci. 9(2), 141-159(2015)

18. Kara, E.E., Ilkhan, M.: Some properties of generalized Fibonacci sequence spaces. Linear Multilinear Algebra 64(11), 2208-2223 (2016)

19. Maddox, I.J.: Spaces of strongly summable sequences. Q. J. Math. 18, 345-355 (1967)

20. Maddox, I.J.: Paranormed sequence spaces generated by infinite matrices. Math. Proc. Camb. Philos. Soc. 64, 335-340 (1968)

21. Maddox, I.J.: Some properties of paranormed sequence spaces. J. Lond. Math. Soc. (2) 1, 316-322 (1969)

22. Malkowsky, E., Rakocevic, V.: On matrix domains of triangles. Appl. Math. Comput. 189(2), 1146-1163 (2007)

23. Mohiuddine, S.A.: Matrix transformations of paranormed sequence spaces through de la Vallée-Pousin mean. Acta Sci., Technol. 37(1), 71-75 (2015)

24. Mohiuddine, S.A., Hazarika, B.: Some classes of ideal convergent sequences and generalized difference matrix operator. Filomat 31(6), 1827-1834 (2017)

25. Mohiuddine, S.A., Raj, K.: Vector valued Orlicz-Lorentz sequence spaces and their operator ideals. J. Nonlinear Sci. Appl. 10, 338-353 (2017)

26. Mohiuddine, S.A., Raj, K., Alotaibi, A.: Generalized spaces of double sequences for Orlicz functions and bounded-regular matrices over n-normed spaces. J. Inequal. Appl. 2014, 332 (2014)

27. Mursaleen, M., Mohiuddine, S.A.: Regularly $\sigma$-conservative and $\sigma$-coercive four dimensional matrices. Comput. Math. Appl. 56(6), 1580-1586 (2008)

28. Mursaleen, M., Mohiuddine, S.A.: On $\sigma$-conservative and boundedly $\sigma$-conservative four-dimensional matrices. Comput. Math. Appl. 59(2), 880-885 (2010)

29. Nakano, H.: Modulared sequence space. Proc. Jpn. Acad., Ser. A, Math. Sci. 27, 508-512 (1951)

30. Sarıgöl, M.A.: On absolute summability factors. Comment. Math. Prace Mat. 31, 157-163 (1991)

31. Sarıgöl, M.A.: On local properties of factored Fourier series. Appl. Math. Comput. 216, 3386-3390 (2010)

32. Sarıgöl, M.A.: Matrix transformations on fields of absolute weighted mean summability. Studia Sci. Math. Hung. 48(3), 331-341 (2011)

33. Sarıgöl, M.A.: An inequality for matrix operators and its applications. J. Class. Anal. 2, 145-150 (2013)

34. Sarıgöl, M.A.: Spaces of series summable by absolute Cesàro and matrix operators. Commun. Math. Appl. 7(1), 11-22 (2016)

35. Sulaiman, W.T.: On summability factors of infinite series. Proc. Am. Math. Soc. 115, 313-317 (1992)

36. Wilansky, A.: Summability Through Functional Analysis. Mathematics Studies, vol. 85. North-Holland, Amsterdam (1984)

37. Yeşilkayagil, M., Başar, F.: On the paranormed Nörlund sequence space of nonabsolute type. Abstr. Appl. Anal. 2014 Article ID 858704 (2014)

\section{Submit your manuscript to a SpringerOpen ${ }^{\circ}$ journal and benefit from:}

- Convenient online submission

- Rigorous peer review

- Open access: articles freely available online

- High visibility within the field

- Retaining the copyright to your article

Submit your next manuscript at $>$ springeropen.com 